\title{
O poziomach sprzeczności zbiorów przesłanek
}

DOI: http://dx.doi.org/10.12775/RF.2016.008

Raymond Jennings i Peter Schotch w artykule Inference and necessity ${ }^{1}$ wprowadzili pojęcie poziomu sprzeczności (niekoherencji) zbioru przesłanek. Miara ta wyznaczona jest przez najmniejszą liczbę podzbiorów, na jakie trzeba podzielić dany zbiór, aby każdy z elementów podziału był niesprzeczny. Idea wykorzystywania właściwych podzbiorów zbioru przesłanek znajduje odzwierciedlenie we wnioskowaniach znanych z życia codziennego, w ramach których nie posługujemy się jednocześnie całą naszą wiedza, lecz jedynie jej fragmentami ${ }^{2}$. Pojęcie poziomu sprzeczności zbioru pozwoliło Jenningsowi i Schotchowi na sformułowanie parakonsystentnej relacji inferencji zwanej forcingiem. O ile klasyczna relacja konsekwencji zachowuje prawdziwość, tak relacja forcingowa zachowuje poziom sprzeczności zbioru przesłanek ${ }^{3}$.

Celem pracy jest omówienie pewnych własności sprzecznych zbiorów przesłanek, które można zaobserwować posługując się metodą wnioskowania zaproponowaną przez Jenningsa i Schotcha. Sformułujemy pojęcia potrzebne do wyrażenia forcingowej relacji konsekwencji. Przedstawimy również przykłady jej zastosowania.

Niech następujące symbole będą jedynymi składowymi alfabetu języka L: p, ', ᄀ, $\Lambda, \rightarrow,($, ). Symbole "p" oraz "'” będą służyły do budowa-

1 P.K. Schotch, R.E. Jennings, Inference and Necessity, "Journal of Philosophical Logic", 1980, nr 9, s. 327-340.

2 Ibidem, s, 329.

$3 \mathrm{~W}$ literaturze anglojęzycznej podejście do parakonsystencji zaproponowane przez Jenningsa i Schotcha określane jest jako preservationism. 
nia zmiennych zdaniowych języka L ${ }^{4}$. Przypominamy poniżej określenia wyrażenia, zbioru zmiennych zdaniowych oraz zbioru formut.

Definicja 1. Każdy skończony ciąg symboli z alfabetu języka L nazywamy wyrażeniem języka L.

Definicja 2. Zbiór zmiennych zdaniowych Zm to najmniejszy spośród zbiorów wyrażeń X języka L spełniający następujące warunki:

$$
\begin{array}{ll}
\text { i. } & p \in X, \\
\text { ii. } & \text { jeżeli }{ }^{\ulcorner} A^{\urcorner} \in X, \text { to }{ }^{\ulcorner} A^{\prime\urcorner} \in X .
\end{array}
$$

Definicja 3. Zbiór formuł For to najmniejszy spośród zbiorów $X$ wyrażeń języka L spełniających następujące warunki:

i. Zm zawarty jest $\mathrm{w} X$,

ii. jeżeli $A \in X$, to ${ }^{r} \neg A^{\urcorner} \in X$,

iii. jeżeli $A, B \in X$, to ${ }^{\ulcorner} A \wedge B^{\urcorner} \in X$,

iv. jeżeli $A, B \in X$, to ${ }^{\top} A \rightarrow B^{\urcorner} \in X$.

Logika L nad językiem L nazywamy dowolny zbiór par $\langle\Gamma, \alpha\rangle \in 2^{\text {For }} \times$ For. Dla dowolnej pary $\langle\Gamma, \alpha\rangle \in \mathbf{L}$, piszemy $\Gamma \vdash_{L} \alpha$. Ponieważ w naszych przykładach jako L bierzemy logike (konsekwencję) klasyczna, wyprowadzenie ze zbioru $\Gamma$ formuły $\alpha$ oznaczamy pisząc $\Gamma \vdash \alpha$. W przypadku rozważań ogólnych, będziemy odnosili się do pewnej ustalonej logiki L. Przyjmujemy, iż relacja $\vdash_{L}$ posiada poniżej przywołane własności określane odpowiednio jako zwrotność, cięcie i monotoniczność:

i. $\quad$ jeżeli $\alpha \in \Gamma$, to $\Gamma \vdash_{L} \alpha$,

ii. $\quad$ jeżeli $\Gamma \cup\{\alpha\} \vdash_{L} \beta$ oraz $\Gamma \vdash_{L} \alpha$, to $\Gamma \vdash_{L} \beta$,

iii. $\quad$ jeżeli $\Gamma \vdash_{L} \alpha$, to $\Gamma \cup \Delta \vdash_{L} \alpha$.

Ponadto, dla dowolnego zbioru $\Gamma$ niech $\operatorname{Card}(\Gamma)$ oznacza jego liczność, czyli moc. Będziemy rozważać zbiory $\Gamma$ spełniające warunek: $\operatorname{Card}(\Gamma)<\aleph_{0}$.

Definicja 4.

1. Zbiór $\Gamma \subsetneq$ For jest niesprzeczny względem logiki L (w skrócie: L-niesprzeczny; ozn. $\left.\operatorname{Con}_{L}(\Gamma)\right)$ wtw $\exists_{\alpha \in \text { For }} \Gamma \nvdash_{L} \alpha$.

4 Dla przejrzystości zapisu pierwsze sześć zmiennych zdaniowych będziemy oznaczać przez ' $p^{\prime},{ }^{\prime} q$ ', ' $r$ ', 's', ' $t$ ', ' $w$ '. 
2. Zbiór formuł $\Gamma \subseteq$ For jest sprzeczny względem logiki L (w skrócie: L-sprzeczny) wtw nie jest L-niesprzeczny ${ }^{5}$, piszemy wówczas: $\overline{\operatorname{Con}_{L}}(\Gamma)$.

Definicja 5. Formuła $\alpha \in$ For jest wewnętrznie sprzeczna względem logiki L (w skrócie: wewnętrznie L-sprzeczna) wtw $\overline{\operatorname{Con}_{L}}(\{\alpha\})$.

Definicja 6. Niech dany będzie zbiór $\Gamma \subsetneq$ For oraz $\mathbb{P}$ - rodzina zbiorów formuł, której jednym z elementów jest zbiór pusty $(\varnothing)$. $\mathbb{P}$ jest logicznym pokryciem zbioru $\Gamma$ ze względu na logikę $\mathbf{L}\left(\operatorname{ozn} . \operatorname{Cov}_{L}(\mathbb{P}, \Gamma)\right)$ wtw zachodzą następujące warunki:

i. $\quad$ dla każdego $\Omega \in \mathbb{P}, \operatorname{Con}_{L}(\Omega)$,

ii. $\quad \Gamma=\bigcup \mathbb{P}$.

Przykład 1. Rozważmy zbiór $\Gamma=\{p, \neg p, p \rightarrow q, p \wedge \neg q, q\}$. Zgodnie $\mathrm{z}$ definicja, jednym z logicznych pokryć zbioru $\Gamma$ jest rodzina:

$$
\begin{aligned}
& \mathbb{P}=\left\{\Omega_{0}, \Omega_{1}, \Omega_{2}, \Omega_{3}\right\}, \text { gdzie } \Omega_{0}=\emptyset, \Omega_{1}=\{p, p \rightarrow q\}, \Omega_{2}= \\
& =\{\neg p, q\}, \Omega_{3}=\{p \wedge \neg q\} .
\end{aligned}
$$

Zbiór pusty jest elementem każdej rodziny zbiorów będącej pokryciem logicznym, ponieważ chcemy uwzględniać przypadek, gdy $\Gamma=\emptyset$. Przyjmujemy umownie, iż podając przykłady pokryć logicznych, będziemy pomijać ich najmniejszy względem inkluzji element, tj. zbiór $\emptyset$.

Definicja 7. Niech dane będą $\mathbb{P}$ oraz $\Gamma$ takie, że $\operatorname{Cov}_{L}(\mathbb{P}, \Gamma)$. Szerokością pokrycia $\mathbb{P}($ ozn. $w(\mathbb{P}))$ nazywamy liczbę $w(\mathbb{P})=\operatorname{Card}(\mathbb{P})-1$.

Zatem dla zbioru $\Gamma$ i pokrycia logicznego $\mathbb{P}$ danych $w$ przykładzie $1, w(\mathbb{P})=3$.

Kluczową rolę $\mathrm{w}$ rozważaniach prowadzonych przez Jenningsa i Schotcha odgrywa funkcja, która dowolnemu zbiorowi formuł $\Gamma \subsetneq$ For przyporządkowuje liczbę naturalną będącą minimalną szerokością pokryć logicznych $\mathbb{P}$ zbioru $\Gamma$ - jeśli owa minimalna wartość istnieje oraz symbol $\infty$ - jeśli nie istnieje. Zatem, rozróżniana jest sprzeczność zbioru przesłanek polegająca albo na występowaniu w zbiorze pojedynczych formuł wewnętrznie sprzecznych, albo na istnieniu podzbioru formuł wzajemnie sprzecznych (którego poszczególne elementy są niesprzeczne) $)^{6}$.

5 Widać, że zbiór nie jest L-niesprzeczny wtw $\forall_{\alpha \in \text { For }} \Gamma \vdash_{L} \alpha$.

6 P.K. Schotch, R.E. Jennings, On Detonating, w: G. Priest, R. Routley, J. Norman (eds.), Paraconsistent Logic: Essays on the Inconsistent, München: Philosophia Verlag, 1989, s. 308. 
Definicja 8. Niech $\ell^{L}: \mathbf{2}^{\text {For }} \rightarrow \mathbb{N} \cup\{\infty\}$ będzie funkcją określoną dla dowolnego $\Gamma \subsetneq$ For następująco:

$$
\begin{gathered}
\rho L(\Gamma)=\left\{\min \left\{w(\mathbb{P}): \mathbb{P} \subsetneq 2^{\text {For }} \operatorname{oraz}^{\text {istnieje }} \operatorname{Cov}_{L}(\mathbb{P}, \Gamma)\right\},\right. \text { o ile owo minimum } \\
\infty, \text { w przeciwnym przypadku }
\end{gathered}
$$

$\ell^{L}(\Gamma)$ nazywamy poziomem sprzeczności zbioru formut $\Gamma$ (ze względu na logikę $\mathbf{L}$ ). Jeżeli funkcja $\ell^{L}$ dla zbioru $\Gamma$ przyjmuje wartości 0 (odpowiednio 1), to $\Gamma=\emptyset$ (odpowiednio $\Gamma$ jest zbiorem L-niesprzecznym $\left.\left(\operatorname{czyli} \operatorname{Con}_{L}(\Gamma)\right)\right)$. W przypadku, gdy $\ell^{L}(\Gamma)=\infty$, zbiór $\Gamma$ zawiera formułę wewnętrznie sprzeczną względem logiki L. Wówczas nie istnieje pokrycie logiczne zbioru $\Gamma$ - żadna rodzina zbiorów nie spełnia jednocześnie warunków i oraz ii definicji 6.

Jeżeli

$\ell^{L}(\Gamma)>1$ i $\ell^{L}(\Gamma) \neq \infty$, to

$\overline{\mathrm{Con}_{L}}(\Gamma)$ oraz $\Gamma$ nie zawiera formuły wewnętrznie sprzecznej. $(*)$

Warunek ( pociaga za sobą to, że albo istnieją formuły $\alpha, \beta \in \Gamma$ takie, że $\overline{\operatorname{Con}_{L}}(\{\alpha, \beta\})$, albo nie ma takich $\alpha, \beta \in \Gamma$, że $\overline{\operatorname{Con}_{L}}(\{\alpha, \beta\})$ oraz $\operatorname{Card}(\Gamma) \geq 3$.

Przykład 2. Rozważmy zbiór $\Gamma=\{p, q, \neg p, p \wedge \neg q, r \wedge s$, $t \wedge \neg r, w, q \wedge t\}$. Niech $\mathbb{P}=\left\{\Omega_{1}, \Omega_{2}\right\}$ będzie pokryciem zbioru $\Gamma$, takim że:

$$
\begin{aligned}
& \Omega_{1}=\{t \wedge \neg r, q \wedge t, p, q\}, \\
& \Omega_{2}=\{r \wedge s, p \wedge \neg q, w, p\} .
\end{aligned}
$$

Zgodnie z definicją $7, w(\mathbb{P})=2$. Ponieważ szerokość pokrycia $\mathbb{P}$ nie może być mniejsza ( $\Gamma$ zawiera formuły parami sprzeczne), $\ell^{L}(\Gamma)=2$.

Przykład 3. Niech dany będzie zbiór $\Gamma=\{p, \neg q, p \rightarrow q\}$. Ponieważ $\overline{\operatorname{Con}_{L}}(\Gamma)$ i $\Gamma$ nie zawiera formuly wewnętrznie sprzecznej, zatem $\ell^{L}(\Gamma)>1$ i $\ell^{L}(\Gamma) \neq \infty$. Niech $\mathbb{P}_{1}=\{\{\neg q\},\{p, p \rightarrow q\}\}$. Stąd, $w\left(\mathbb{P}_{1}\right)=2$. Skoro szerokość pokrycia zbioru $\Gamma$ nie może być mniejsza, zatem $\ell^{L}(\Gamma)=2$.

Definicja 9. Niech $\mathcal{P}_{\Gamma}=\left\{\mathbb{P} \subsetneq 2^{\text {For }}: \operatorname{Cov}_{L}(\mathbb{P}, \Gamma)\right.$ oraz $\operatorname{Card}(\mathbb{P})=$ $\left.=\ell^{L}(\Gamma)+1\right\} . \mathcal{P}_{\Gamma}$ nazywamy zbiorem minimalnych logicznych pokryć zbioru formut $\Gamma$. 
Zbiór $\mathcal{P}_{\Gamma}$ jest niepusty, gdy dla $\Gamma \subsetneq$ For, $\ell^{L}(\Gamma) \neq \infty$. Natomiast w przypadku, gdy $\ell^{L}(\Gamma)=0$ lub $\ell^{L}(\Gamma)=1$, to $\operatorname{Card}\left(\mathcal{P}_{\Gamma}\right)=1$. Z kolei, jeżeli $\ell^{L}(\Gamma)>1$ i $\ell^{L}(\Gamma) \neq \infty$, to $\operatorname{Card}\left(\mathcal{P}_{\Gamma}\right) \geq 1$.

Dla zbioru $\Gamma$ z przykładu 3, $\operatorname{Card}\left(\mathcal{P}_{\Gamma}\right)=3$. Zbiór $\mathcal{P}_{\Gamma}$ składa się z następujących pokryć logicznych?

$$
\begin{aligned}
& \mathbb{P}_{1}=\{\varnothing,\{\neg q\},\{p, p \rightarrow q\}\}, \\
& \mathbb{P}_{2}=\{\varnothing,\{p, \neg q\},\{p \rightarrow q\}\}, \\
& \mathbb{P}_{3}=\{\varnothing,\{p\},\{p \rightarrow q, \neg q\}\} .
\end{aligned}
$$

Dla dowolnego zbioru $\Gamma \subsetneq$ For takiego, że $\ell^{L}(\Gamma) \neq \infty$ wprowadzamy relację inferencji nazywaną forcingiem.

Definicja 10. $\Gamma \Vdash \alpha$ wtw dla każdego $\mathbb{P} \in \mathcal{P}_{\Gamma}$ istnieje $\Omega \in \mathbb{P}$ takie, że $\Omega \vdash_{L} \alpha$.

W przypadku, gdy wartość funkcji $\ell^{L}(\Gamma)$ wynosi 0 lub 1 , tj. gdy wnioskujemy odpowiednio ze zbioru pustego lub niepustego zbioru $\Gamma$-niesprzecznego, forcing zachowuje się jak relacja $\vdash_{L}$. Stąd, w dalszych rozważaniach skupimy się na analizie przypadków zbiorów, których poziom sprzeczności jest większy od 1 (i różny od $\infty$ ).

Przykład 4. Rozważmy następujący zbiór $\Gamma=\{\neg r \wedge \neg S, r \wedge p$, $s \wedge(p \rightarrow q), t\}$. Do zbioru $\mathcal{P}_{\Gamma}$ należą dwa pokrycia logiczne:

$$
\begin{aligned}
& \mathbb{P}_{1}=\left\{\Omega_{1}, \Omega_{2}\right\}, \text { gdzie } \Omega_{1}=\{r \wedge p, s \wedge(p \rightarrow q), t\}, \Omega_{2}=\{\neg r \wedge \neg s\}, \\
& \mathbb{P}_{2}=\left\{\Omega_{1}, \Omega_{2}\right\}, \text { gdzie } \Omega_{1}=\{r \wedge p, s \wedge(p \rightarrow q)\}, \Omega_{2}=\{\neg r \wedge \neg s, t\} .
\end{aligned}
$$

Zatem $\Gamma \Vdash q$, ponieważ dla każdego pokrycia logicznego istnieje zbiór $\Omega$ taki, że $\Omega \vdash q$, tj. dla obu pokryć jest to $\Omega_{1}$.

Fakt 1. Dla dowolnego zbioru $\Gamma$, takiego że $\Gamma \subsetneq$ For, $\ell^{L}(\Gamma)>$ $>1$ i $\ell^{L}(\Gamma) \neq \infty$, oraz dowolnej formuły $\alpha$ zachodzi: jeżeli $\Gamma \Vdash \alpha$, to $\ell^{L}(\Gamma)=\ell^{L}(\Gamma \cup\{\alpha\})$.

Dowód. Załóżmy, że $\ell^{L}(\Gamma)=k$ oraz $\Gamma \Vdash \alpha$. Niech $\mathbb{P}$ będzie dowolnym pokryciem należącym do $\mathcal{P}_{\Gamma}$. Na mocy definicji 10, dla co najmniej jednego $\Omega \in \mathbb{P}, \Omega \vdash_{L} \alpha$. Dodajmy formułę $\alpha$ do wszystkich niepustych elementów pokrycia $\mathbb{P}, \mathrm{z}$ których można tę formułę wyprowadzić. Otrzymujemy w ten sposób pokrycie logiczne zbioru $\Gamma \cup\{\alpha\}$, gdyż ze

$7 \mathrm{~W}$ celu pełnego przedstawienia zbioru $\mathcal{P}_{\Gamma}$ zawieszamy w tym momencie przyjętą uprzednio umowę dotyczącą pomijania pustego elementu pokrycia logicznego. 
względu na założenie oraz warunek cięcia dla relacji $\vdash_{L}$, rozszerzenia zbiorów należących do pokrycia $\mathbb{P}$ są niesprzeczne względem logiki $\mathbf{L}$. Ponadto konstrukcja gwarantuje, iż szerokość otrzymanego pokrycia jest równa $k$. Zatem minimalna szerokość pokrycia logicznego zbioru $\Gamma \cup\{\alpha\}$ jest niewiększa niż $k$, czyli $\ell^{L}(\Gamma \cup\{\alpha\}) \leq k$.

Przyjmijmy, że $\ell^{L}(\Gamma \cup\{\alpha\})=l<k$. Rozważmy dowolne pokrycie logiczne zbioru $\Gamma \cup\{\alpha\}$. Na mocy definicji 6 formuła $\alpha$ jest elementem co najmniej jednego $\Omega$ należącego do tego pokrycia. Ze wszystkich takich zbiorów $\Omega$ usuwamy formułę $\alpha$, otrzymując w ten sposób pokrycie logiczne zbioru $\Gamma$, którego szerokość jest mniejsza lub równa $l$ (jest mniejsza, gdy jeden z elementów pokrycia ma postać $\{\alpha\}$ ). Lecz jest to sprzeczne z założeniem, że $\ell^{L}(\Gamma)=k$. Stąd wnosimy, iż $\ell^{L}(\Gamma \cup\{\alpha\})=k$.

Fakt 2. Dla każdego zbioru $\Gamma \subsetneq$ For, jeżeli $\alpha \in \Gamma$, to $\Gamma \Vdash \alpha$.

Dowód. Niech $\Gamma$ będzie zbiorem, dla którego $\ell^{L}(\Gamma)=\infty$. Wówczas zbiór $\mathcal{P}_{\Gamma}$ jest pusty, a zatem na mocy definicji 10, teza zachodzi w sposób trywialny. Załóżmy, że $\Gamma$ jest zbiorem, dla którego $\ell^{L}(\Gamma) \neq \infty$. Niech $\mathbb{P}$ będzie dowolnym pokryciem należącym do $\mathcal{P}_{\Gamma}$. Z warunku 2 definicji 6 wiemy, iż $\Gamma=\bigcup \mathbb{P}$. Czyli każda formuła $\alpha \in \Gamma$ jest elementem pewnego $\Omega \in \mathbb{P}$. Zatem, na mocy zwrotności relacji $\vdash_{L}$, dla każdej formuly $\alpha \in \Gamma$ istnieje takie $\Omega \in \mathbb{P}$, że $\Omega \vdash_{L} \alpha$.

Tym samym pokazaliśmy, że relacja ॥ spełnia warunek zwrotności.

Fakt 3. Dla każdego zbioru $\Gamma \subsetneq$ For, który spełnia warunek $\ell^{L}(\Gamma)>1$ i $\ell^{L}(\Gamma) \neq \infty$, oraz dowolnych $\alpha, \beta \in$ For, jeżeli $\Gamma \cup\{\alpha\} \Vdash \beta$ oraz $\Gamma \Vdash \alpha$, to $\Gamma \Vdash \beta$.

Dowód. Jeśli $\alpha \in \Gamma$, teza zachodzi w sposób trywialny. Załóżmy więc, że (1) $\Gamma \cup\{\alpha\} \Vdash \beta$, (2) $\Gamma \Vdash \alpha, \alpha \notin \Gamma$ oraz $\ell^{L}(\Gamma)=k$. Niech $\mathbb{P} \in \mathcal{P}_{\Gamma}$. Na mocy (2) i definicji 10 istnieje $\Omega \in \mathbb{P}$ takie, że $\Omega \vdash_{L} \alpha$. Z pokrycia logicznego $\mathbb{P}$, tworzymy rodzinę $\mathbb{P}_{\alpha}$ przez dołączenie formuły $\alpha$ do każdego elementu $\Omega$ pokrycia $\mathbb{P}$ takiego, że $\Omega \vdash_{L} \alpha$ oraz pozostawienie innych elementów rodziny $\mathbb{P}$ bez zmian. Opisaną procedurę powtarzamy dla każdego elementu $\mathbb{P} \in \mathcal{P}_{\Gamma}$. Oznaczmy zbiór tak otrzymanych rodzin przez $\mathcal{P}_{\Gamma}^{\alpha}$. Z faktu 1 elementy $\mathcal{P}_{\Gamma}^{\alpha}$ są pokryciami logicznymi zbioru $\Gamma \cup\{\alpha\}$. Sposób konstrukcji gwarantuje, iż dla każ$\operatorname{dego} \mathbb{P} \in \mathcal{P}_{\Gamma}, \mathrm{w}(\mathbb{P})=\mathrm{w}\left(\mathbb{P}_{\alpha}\right)=k$. Ponownie na mocy faktu 1 wiemy, że $\ell^{L}(\Gamma)=\ell^{L}(\Gamma \cup\{\alpha\})$. Stąd wnosimy, iż $\mathcal{P}_{\Gamma}^{\alpha} \subseteq \mathcal{P}_{\Gamma \cup\{\alpha\}}$.

Weźmy dowolne $\mathbb{P} \in \mathcal{P}_{\Gamma}$ i rozważmy jego modyfikację $\mathbb{P}_{\alpha} \in \mathcal{P}_{\Gamma}^{\alpha}$. $\mathrm{Z}$ założenia (1) wiemy, iż dla każdego minimalnego pokrycia zbioru $\Gamma \cup\{\alpha\}$ istnieje taki jego element $\Omega$, że $\Omega \vdash_{L} \beta$. Ponieważ $\mathcal{P}_{\Gamma}^{\alpha} \subseteq \mathcal{P}_{\Gamma \cup\{\alpha\},}$ więc w szczególności dla pokrycia $\mathbb{P}_{\alpha}$ istnieje $\Omega \in \mathbb{P}_{\alpha}$ taki, że $\Omega \vdash_{L} \beta$ oznaczmy ten zbiór jako $\Omega_{\beta}$. Należy rozważyć dwie możliwości: 
albo $\alpha \in \Omega_{\beta}$ albo $\alpha \notin \Omega_{\beta}$. Przyjmijmy, że zachodzi pierwsza z nich, tj. $\alpha \in \Omega_{\beta}$. Ze względu na sposób konstrukcji pokrycia $\mathbb{P}_{\alpha}$ otrzymujemy wówczas, iż $\Omega_{\beta}=\Omega \cup\{\alpha\}$, dla pewnego $\Omega \in \mathbb{P}$ takiego, że $\Omega \vdash_{L} \alpha$. Stąd na mocy reguty cięcia dla relacji inferencji $\vdash_{L}$, skoro $\Omega \cup\{\alpha\} \vdash_{L} \beta$ oraz $\Omega \vdash_{L} \alpha$, to $\Omega \vdash_{L} \beta$.

Załóżmy, że $\alpha \notin \Omega_{\beta}$. Zatem $\Omega_{\beta} \in \mathbb{P}$, ponieważ przy przekształcaniu pokrycia $\mathbb{P} w \mathbb{P}_{\alpha}$, zbiór $\Omega_{\beta}$ pozostał niezmieniony. Czyli istnieje element $\Omega$ pokrycia $\mathbb{P}$ (jest nim $\Omega_{\beta}$ ) taki, że $\Omega \vdash_{L} \beta$. Ze względu na dowolność $\mathbb{P}$ otrzymujemy, iż $\Gamma \Vdash \beta$.

Pokazaliśmy, iż forcing spełnia regułe cięcia. Kolejną własnością do rozważenia jest monotoniczność.

Przykład 5. Niech dany będzie zbiór $\Gamma=\{p \wedge q, \neg q, q \wedge r\}$. Poziom sprzeczności tego zbioru wyznaczony przez wartość funkcji $\ell^{L}(\Gamma)$ wynosi 2. Zbiór $\mathcal{P}_{\Gamma}$ ma jeden element:

$$
\mathbb{P}=\left\{\Omega_{1}, \Omega_{2}\right\}, \text { gdzie } \Omega_{1}=\{p \wedge q, q \wedge r\}, \Omega_{2}=\{\neg q\} .
$$

Widzimy, iż $\Gamma \Vdash p \wedge r$, ponieważ istnieje element $\Omega$ taki, że $\Omega \vdash p \wedge r$.

Rozważmy zbiór $\quad \Gamma^{\prime}=\Gamma \cup\{q \wedge \neg r\}=\{p \wedge q, \neg q, q \wedge r, q \wedge \neg r\}$. Mamy dokładnie dwa elementy zbioru $\mathcal{P}_{\Gamma}$ :

$\mathbb{P}_{1}=\left\{\Omega_{1}, \Omega_{2}, \Omega_{3}\right\}$, gdzie $\Omega_{1}=\{p \wedge q, q \wedge r\}, \Omega_{2}=\{\neg q\}, \Omega_{3}=\{q \wedge \neg r\}$

oraz

$$
\begin{aligned}
& \mathbb{P}_{2}=\left\{\Omega_{1}, \Omega_{2}, \Omega_{3}\right\}, \text { gdzie } \Omega_{1}=\{p \wedge q, q \wedge \neg r\}, \Omega_{2}= \\
& =\{\neg q\}, \Omega_{3}=\{q \wedge r\} .
\end{aligned}
$$

Widzimy, iż nie dla każdego elementu zbioru $\mathcal{P}_{\Gamma}$ istnieje $\Omega \in \mathbb{P}$ takie, że $\Omega \vdash p \wedge r$. Zatem zwiększenie poziomu sprzeczności zbioru formuł może powodować rozdzielenie pewnych układów przesłanek (znajdować się będą w różnych elementach pokrycia), które uniemożliwi wyprowadzenie uprzednio uzyskiwanych konkluzji. Stąd otrzymujemy wniosek: forcing nie spełnia warunku monotoniczności.

Jennings i Schotch podkreślają jednak, iż proponowana przez nich relacja inferencji posiada własność monotoniczności w ograniczonym wariancie ${ }^{8}$.

\footnotetext{
8 G. Payette, P.K. Schotch, On Preserving, „Logica Universalis”, 2007, s. 295-310.
} 
Fakt 4. Dla każdego zbioru $\Gamma \subsetneq$ For, który spełnia $\ell^{L}(\Gamma)>1$ i $\ell^{L}(\Gamma) \neq \infty$ oraz dowolnych $\alpha, \beta \in$ For, jeżeli $\Gamma \Vdash \alpha$ oraz $\ell^{L}(\Gamma)=\ell^{L}(\Gamma \cup\{\beta\}), \Gamma \cup\{\beta\} \Vdash \alpha$.

Dowód. Załóżmy, że $\Gamma \Vdash \alpha$ oraz $\ell^{L}(\Gamma)=\ell^{L}(\Gamma \cup\{\beta\})$. Niech dany będzie zbiór minimalnych pokryć $\mathcal{P}_{\Gamma \cup\{\beta\}}$ oraz dowolny jego element $\mathbb{P}$. $Z$ definicji 6 wiemy, iż dla co najmniej jednego $\Omega \in \mathbb{P}, \beta \in \Omega$. Bierzemy kolejno elementy $\mathbb{P}$ i modyfikujemy w następujący sposób: dla każdego $\Omega$ takiego, że $\beta \in \Omega$ przyjmujemy, że $\Omega^{\backslash \beta}=\Omega \backslash\{\beta\}$, w pozostałych przypadkach, czyli dla $\Omega$ takich, że $\beta \notin \Omega, \Omega$ pozostawiamy bez zmian. Stosując tę metodę przekształcamy $\mathbb{P}$ w zbiór $\mathbb{P}^{\prime}$. Zgodnie z przyjętym założeniem otrzymujemy $\mathrm{w}$ ten sposób pokrycie zbioru $\Gamma$ takie, że $\mathrm{w}\left(\mathbb{P}^{\prime}\right)=\ell^{L}(\Gamma \cup\{\beta\})$. Ponieważ z założenia, dla każdego $\mathbb{P} \in \mathcal{P}_{\Gamma}$ istnieje $\Omega \in \mathbb{P}$, takie że $\Omega \vdash_{L} \alpha$, zatem również jest tak dla $\mathbb{P}^{\prime}$. Niech $\Omega^{\prime}$ będzie takim elementem pokrycia $\mathbb{P}^{\prime}$, że $\Omega^{\prime} \vdash_{L} \alpha$. Ze względu na konstrukcję pokrycia $\mathbb{P}^{\prime}$ rozważmy dwa przypadki: albo (1) $\Omega^{\prime}=\Omega^{\backslash \beta}$, dla pewnego $\Omega \in \mathbb{P}$ takiego, że $\beta \in \Omega$, albo (2) $\Omega^{\prime}=\Omega$, dla pewnego $\Omega \in \mathbb{P}$ takiego, że $\beta \notin \Omega$. Jeżeli zachodzi (1), to na mocy monotoniczności relacji $\vdash_{L}$ mamy: $\Omega^{\prime} \cup\{\beta\} \vdash_{L} \alpha$. Lecz $\Omega^{\prime} \cup\{\beta\}=\Omega$, czyli $\Omega \vdash_{L} \alpha$, dla $\Omega \in \mathbb{P}$ wskazanego w (1). Jeżeli zachodzi zaś (2), to również $\Omega \vdash_{L} \alpha$, dla pewnego $\Omega \in \mathbb{P}$.

Posługując się forcingiem sprowadzamy konsekwencję L-sprzecznego zbioru przesłanek $\Gamma$ do konsekwencji pewnych jego L-niesprzecznych podzbiorów wyznaczonych przez wartość funkcji $\ell^{L}(\Gamma)$ określającą poziom sprzeczności danego zbioru. Zauważmy, że definicja 10 głosi, iż wnioskiem uzyskanym przy użyciu forcingowej relacji inferencji może być tylko taka formuła, która jest wyprowadzalna z przesłanek należących do jakiegoś elementu pokrycia $\mathbb{P}$. Jednakże, definicja 6 pokrycia logicznego wymusza jedynie, by jego elementy były L-niesprzeczne oraz by ich suma była równa zbiorowi wyjściowemu. Zatem wśród wszystkich dostępnych pokryć logicznych przykładowo mogą być te, których elementy są maksymalnie L-niesprzecznymi podzbiorami zbioru $\Gamma$ ( $\mathfrak{j}$. takimi, które oprócz warunków definicji 6 spełniają wymóg, że każdy ich właściwy nadzbiór zawarty w $\Gamma$ jest L-sprzeczny), jak i te, których elementy są zbiorami parami rozłącznymi. Taka dowolność formowania pokryć logicznych powoduje, iż pewne klasyczne konsekwencje niesprzecznych podzbiorów rozważanego zbioru przesłanek (podzbiorów, a nie elementów pokryć logicznych) będą niemożliwe do uzyskania jako forcingowe konsekwencje.

Rozważmy następujący przykład.

Przykład 6. Niech $\Gamma=\{p, \neg r, r, p \rightarrow q\}$. Mamy wówczas następujące trzy pokrycia: 


$$
\begin{aligned}
& \mathbb{P}_{1}=\{\{p, r, p \rightarrow q\},\{\neg r\}\}, \\
& \mathbb{P}_{2}=\{\{p, r\},\{\neg r, p \rightarrow q\}\}, \\
& \mathbb{P}_{3}=\{\{p, \neg r, p \rightarrow q\},\{r\}\} .
\end{aligned}
$$

Pomimo, że przesłanki $\{p, p \rightarrow q\} \subsetneq \Gamma$ nie odgrywają roli w usprzecznianiu zbioru $\Gamma$, to nie pozwalają na uzyskanie formuły $q$ jako wniosku. Taki stan, powołując się na słowa Grahama Priesta9 ${ }^{9}$ można uznać za intuicyjnie błędny.

Spójrzmy na rozważaną metodę wnioskowania z punktu widzenia „wyodrębniania" przesłanek, z których wnioskowanie ma nastąpići ${ }^{10}$. Sposób ten może wyrazić następująco: „,szukajmy takich układów przesłanek, które przy tworzeniu minimalnych pokryć logicznych zachowują «pewną» regularność".

Przykład 7. W przykładzie 6 nie mogliśmy wyprowadzić wniosku $q$, ponieważ przesłanki potrzebne do jego wyprowadzenia nie należały do tego samego elementu pokrycia logicznego. Jednak dla zbioru $\Gamma$ mającego postać: $\Gamma=\{p \wedge \neg r, p \wedge r, p \rightarrow q\}$ otrzymujemy następujące pokrycia logiczne:

$$
\begin{aligned}
& \mathbb{P}_{1}=\left\{\Omega_{1}, \Omega_{2}\right\}, \text { gdzie } \Omega_{1}=\{p \wedge r, p \rightarrow q\}, \Omega_{2}=\{p \wedge \neg r\}, \\
& \mathbb{P}_{2}=\left\{\Omega_{1}, \Omega_{2}\right\}, \text { gdzie } \Omega_{1}=\{p \wedge r\}, \Omega_{2}=\{p \wedge \neg r, p \rightarrow q\} .
\end{aligned}
$$

Tym razem $\Gamma \Vdash q$. Przesłanki $\neg r$ i $r$ wymuszające w przykładzie 6 poziom sprzeczności równy 2 , zostały obie wzmocnione o czynnik $p$, czyli poprzednik implikacji pozwalającej na uzyskanie wniosku $q$.

Przykład 8. Rozważmy zbiór $\Gamma=\{p \wedge q, \neg p \wedge s \wedge r, \neg s \wedge \neg q \wedge r, \neg r, w, s, t\}$.

Poziom sprzeczności tego zbioru wyznaczony przez wartość funkcji $\ell^{L}(\Gamma)$ wynosi 3. Do zbioru $\mathcal{P}_{\Gamma}$ należą m.in. pokrycia:

$$
\begin{aligned}
& \mathbb{P}_{1}=\left\{\Omega_{1}, \Omega_{2}, \Omega_{3}\right\}, \text { gdzie } \Omega_{1}=\{p \wedge q, \neg r, s\}, \Omega_{2}= \\
& =\{\neg p \wedge s \wedge r, w, t\}, \Omega_{3}=\{\neg s \wedge \neg q \wedge r\},
\end{aligned}
$$

9 G. Priest, Paraconsistent Logic, w: Handbook of Philosophical Logic, Vol. 6, D. Gabbay, F. Guenthner (eds.), Dordrecht: Kluwer Academic Publishers, 2002, s. 301.

10 Pomijamy przypadek wyprowadzania konsekwencji z singletonów zbioru przesłanek. 


$$
\begin{aligned}
& \mathbb{P}_{2}=\left\{\Omega_{1}, \Omega_{2}, \Omega_{3}\right\}, \text { gdzie } \Omega_{1}=\{p \wedge q, \neg r, w\}, \Omega_{2}= \\
& =\{\neg p \wedge s \wedge r, s\}, \Omega_{3}=\{\neg s \wedge \neg q \wedge r, t\}, \\
& \mathbb{P}_{3}=\left\{\Omega_{1}, \Omega_{2}, \Omega_{3}\right\}, \text { gdzie } \Omega_{1}=\{p \wedge q, \neg r, t\}, \Omega_{2}= \\
& =\{\neg p \wedge s \wedge r, s\}, \Omega_{3}=\{\neg s \wedge \neg q \wedge r, w\} .
\end{aligned}
$$

W tych trzech przypadkach widać, iż pewne formuły zachowują się regularnie przy zmianie jednego pokrycia logicznego na inne: $\{p \wedge q, \neg r\} \subsetneq \Omega_{1}$ dla każdego z przytoczonych pokryć. Zauważmy również, że formuły $S, W, t$ nie tworzą żadnych stałych układów przesłanek z innymi formułami.

Formuła $\alpha \in$ For jest forcingowa konsekwencją zbioru $\Gamma$, gdy dla każdego pokrycia logicznego $\mathbb{P} \in \mathcal{P}_{\Gamma}$ istnieje jego element $\Omega$ zawierający przesłanki potrzebne do wyprowadzenia formuły $\alpha$. Zatem istnienie przesłanek stanowiących uzasadnienie danej konkluzji ma być tym, co wspólne dla wszystkich pokryć logicznych danego zbioru formuł.

Definicja 11. Niech dany będzie zbiór $\Gamma \subsetneq$ For oraz niepusty zbiór jego minimalnych pokryć logicznych $\mathcal{P}_{\Gamma}{ }^{11}$. Const $(\Gamma)$ nazywamy zbiorem statych elementów pokryć logicznych zbioru $\Gamma$ :

$$
\text { Const }(\Gamma)=\left\{\Delta \in 2^{\Gamma}: \forall_{\mathbb{P} \in \mathcal{P}_{\Gamma}} \exists_{\Omega \in \mathbb{P}} \Delta \subseteq \Omega\right\}
$$

Zbiór Const $(\Gamma)$ jest niepusty, ponieważ na mocy drugiego warunku definicji 6, singletony zbioru $\Gamma$ są elementami Const $(\Gamma)$.

Aby przekonać się, czy zaproponowana definicja zbioru statych elementów pokryć logicznych właściwie oddaje „wyodrębnianie” przesłanek, z których wnioskowanie ma nastąpić, rozważmy następującą hipotezę: zbiór wniosków wyprowadzonych zgodnie z relacją forcingu równy jest zbiorowi wniosków wyprowadzonych klasycznie ze zbioru Const $(\Gamma)$.

Hipoteza: Niech dany będzie zbiór formuł $\Gamma \subsetneq$ For taki, że $\ell^{L}(\Gamma)>1$ i $\ell^{L}(\Gamma) \neq \infty$. Zachodzi wówczas: $\{\alpha \in$ For $: \Gamma \Vdash \alpha\}=\left\{\alpha \in\right.$ For : $\left.\exists_{\Delta \in \operatorname{Const}(\Gamma)} \Delta \vdash \alpha\right\}$.

Inkluzja „,z prawej do lewej” zachodzi w sposób oczywisty. Inkluzja „z lewej do prawej” jest fałszywa, co pokażemy na następującym przykładzie.

Przykład 9. Niech $\Gamma=\{\neg s \wedge p, p \rightarrow q, s \wedge p\}$. Do zbioru $\mathcal{P}_{\Gamma}$ należą dwa pokrycia logiczne:

${ }_{11} \mathcal{P}_{\Gamma} \neq \emptyset$ gwarantuje, iż zbiór $\Gamma$ nie zawiera formuły wewnętrznie sprzecznej. 


$$
\begin{aligned}
& \mathbb{P}_{1}=\left\{\Omega_{1}, \Omega_{2}\right\}, \text { gdzie } \Omega_{1}=\{s \wedge p, p \rightarrow q\}, \Omega_{2}=\{\neg s \wedge p\}, \\
& \mathbb{P}_{2}=\left\{\Omega_{1}, \Omega_{2}\right\}, \text { gdzie } \Omega_{1}=\{\neg s \wedge p, p \rightarrow q\}, \Omega_{2}=\{s \wedge p\}
\end{aligned}
$$

Dla każdego pokrycia $\mathbb{P}$ istnieje $\Omega \in \mathbb{P}$ taka, że $\Omega \vdash q$, czyli $\Gamma \Vdash q$. Natomiast zbiór stałych elementów pokrycia logicznego $\Gamma$ ma postać:

$$
\text { Const }(\Gamma)=\{\{\neg s \wedge p\},\{p \rightarrow q\},\{s \wedge p\}\}
$$

Formuła $q$ nie jest klasyczną konsekwencją żadnego spośród stałych elementów zbioru $\Gamma$.

Zaproponowana definicja 11 okazała się być nieadekwatna względem relacji forcingowej. Jest tak ze względu na utożsamienie statych elementów pokryć logicznych z podzbiorami elementów pokryć logicznych. Niewątpliwie, jak pokazuje przykład 8, posługiwanie się nową definicją pozwala na wyróżnienie niektórych stałych układów formuł występujących w pokryciach. Kwestia istnienia takich stałych elementów dla sprzecznych zbiorów przesłanek wydaje się być warta uwagi. Kluczowe dla ich ujawnienia jest posługiwanie się pojęciem poziomu sprzeczności zbioru, tj. spełnianie warunku by liczba podzbiorów, na jakie dzielimy sprzeczny zbiór przesłanek, była najmniejsza z możliwych.

\section{Bibliografia}

Payette G., Schotch P.K., On Preserving, „Logica Universalis” 2007, s. 295-310 .

Priest G., Paraconsistent Logic, w: Handbook of Philosophical Logic, Vol. 6, D. Gabbay, F. Guenthner (eds.), Dordrecht: Kluwer Academic Publishers, 2002, s. 287-302.

Schotch P.K., Jennings R.E., Inference and Necessity, ,,Journal of Philosophical Logic" 1980, nr 9, s. 327-340.

Schotch P.K., Jennings R.E., On Detonating, w: G. Priest, R. Routley, J. Norman (eds.), Paraconsistent Logic: Essays on the Inconsistent, München: Philosophia Verlag, 1989, s. 306-327.

\section{O poziomach sprzeczności zbiorów przesłanek}

\section{Słowa kluczowe}

Prezerwacjonizm; sprzeczność; Jennings; Schotch; niekoherencja; poziom sprzeczności 
Jedno z podejść do zagadnienia parakonsystencji zostało zapoczątkowane pracami Raymonda Jenningsa i Petera Schotcha, którzy w artykule „Inference and necessity" (1980) zaproponowali pojęcie poziomu sprzeczności (niekoherencji) zbioru przesłanek. Poziom ten jest miarą określającą minimalną liczbę niesprzecznych podzbiorów na jakie można podzielić dany zbiór przesłanek. Pojęcie to pozwoliło na sformułowanie parakonsystentnej relacji inferencji nazywanej forcingiem. Podobnie jak klasyczna relacja inferencji zachowuje prawdziwość, tak relacja forcingowa zachowuje poziom sprzeczności. Celem pracy jest przedyskutowanie przykładów sprzecznych zbiorów przesłanek, które mogą być analizowane przy użyciu metody Jenningsa i Schotcha, przedstawienie uproszczonej (względem oryginalnego sformułowania) wersji podstawowych pojęć potrzebnych do zdefiniowania forcingu oraz pokazanie przykładów użycia tej relacji inferencji.

\section{On levels of inconsistency of sets of premises}

\section{Keywords}

Preservationism; inconsistency; Jennings; Schotch; incoherence; level of inconsistency

A certain approach to paraconsistency was initiated by works of R. Jennings and P. Schotch. In their "Inference and necessity"” (1980) they proposed a notion of a level of inconsistency (incoherence) of a given set of premises. This level is a measure that assigns to a given set of premises $X$, the least number of elements of covers of $X$, that consist of consistent subsets of $X$. The idea of the level of inconsistency allows to formulate a paraconsistent inference relation called by the authors forcing. The proposed approach is known as preservationism. Similarly as classical inference relation is truth-preserving, forcing inference relation is preserving the level of inconsistency. The aim of the paper is to discuss some examples of inconsistent sets of premises, which can be analyzed by the use of Jennings-Schotch's inference method. We will give simplified, with respect to its original formulation, versions of elementary notions needed to define the forcing relation. We also give examples of the use of this inference relation. 